\title{
Efeito da Idade no Afilamento e Sortimento em Povoamentos de Araucaria angustifolia
}

\author{
Afonso Figueiredo Filho' ${ }^{1}$, Fabiane Aparecida de Souza Retslaff ${ }^{1}$, \\ Sintia Valerio Kohler ${ }^{2}$, Marcelo Becker ${ }^{1}$, Diego Brandes ${ }^{1}$ \\ ${ }^{1}$ Departamento de Engenharia Florestal, Universidade Estadual \\ do Centro-Oeste - UNICENTRO, Irati/PR, Brasil \\ ${ }^{2}$ Departamento de Engenharia Florestal, Universidade Federal do Paraná - UFPR, Curitiba/PR, Brasil
}

\begin{abstract}
RESUMO
O objetivo desta pesquisa foi avaliar o efeito da idade no ajuste de funções de afilamento e na evolução do sortimento em árvores masculinas e femininas em plantios de Araucaria angustifolia. Foram amostradas 60 árvores da espécie em um plantio localizado no município de Laranjeiras do Sul, Paraná. Ajustou-se o polinômio de quinto grau para o total de dados, estratificando-os em classes de idade. Foi analisada a evolução do afilamento e do sortimento das árvores com a idade. Os resultados mostraram uma leve vantagem no uso de equações ajustadas por classes de idade e uma melhoria significativa na forma do tronco com o aumento da idade. As árvores femininas $\left(0,9975 \mathrm{~m}^{3}\right)$ apresentaram crescimento mais elevado que as masculinas $\left(0,9384 \mathrm{~m}^{3}\right)$ aos 30 anos. Volumes para os sortimentos mais nobres são produzidos somente a partir da classe de idade 11 a 15 anos.
\end{abstract}

Palavras-chave: Floresta Ombrófila Mista, pinheiro do Paraná, equação de forma.

\section{Effect of Age on Tapering and Assortment in Araucaria angustifolia Stands}

\begin{abstract}
The objective of this research was to evaluate the effect of age on adjust taper functions and evolution of the assortment of male and female trees of Araucaria angustifolia stands. Sixty trees were sampled in a plantation located in the municipality of Laranjeiras do Sul, Paraná state, Brazil. The fifth degree polynomials were adjusted to the whole data set, and they were stratified into age classes. The evolution of the tapering and assortment of trees was analyzed with age. The results showed a slight advantage in the use of equations adjusted for age classes and a significant improvement in the stem form with increasing age. The female trees presented higher growth $\left(0.9975 \mathrm{~m}^{3}\right)$ than the male trees $\left(0.9384 \mathrm{~m}^{3}\right)$ at 30 years of age. Volumes for the noblest assortments are produced only when the stand reaches the age class of 11 to 15 years.
\end{abstract}

Keywords: Mixed Ombrophilous Forest, Parana pine, taper equation. 


\section{INTRODUÇÃO}

A Araucaria angustifolia pertence à família das Araucariáceas, sendo uma espécie dióica, apresentando árvores de sexo masculino e de sexo feminino (Zanon, 2007). A espécie necessita de solos férteis para o seu desenvolvimento, atingindo um bom crescimento em média com 50 anos (Handro \& Ferreira, 1986; Reitz \& Klein, 1966).

A madeira de araucária possui excelente qualidade e se presta a uma infinidade de utilidades, fora o aproveitamento de outras partes da árvore além do tronco, como os nós, resinas e o pinhão. Assim, a alta qualidade da madeira e a grande disponibilidade e demanda da Araucaria angustifolia provenientes das Florestas Ombrófilas Mistas, no Sul do Brasil, foram fatores importantes que contribuíram para a drástica redução das Florestas com Araucária (Sanquetta et al., 2002). Atualmente, a principal fonte de madeira de Araucaria angustifolia que se encontra no mercado provém de plantios.

Segundo Husch et al. (2003), a forma do fuste é definida pela taxa de decréscimo do diâmetro $(d)$, em relação ao diâmetro à altura do peito (DAP), ao longo do tronco. No Brasil, essa relação denomina-se afilamento do fuste (Môra, 2011). Estudos relacionados à forma são baseados em equações que permitem estimar com uma certa acuracidade o diâmetro em qualquer altura do fuste, a partir de variáveis como o DAP (diâmetro a 1,30 metro do solo) e a altura total (Campos \& Leite, 2006).

Essas funções, devido à sua flexibilidade, possibilitam uma estratificação das toras, ou seja, a quantificação dos múltiplos produtos existentes por meio de dimensões pré-estabelecidas (Queiroz et al., 2008). O estudo da forma da árvore é importante no processo de produção florestal, sendo fundamental para a determinação e otimização dos sortimentos da árvore e do povoamento. Para proporcionar o uso múltiplo do tronco de uma árvore em povoamentos florestais é necessário o conhecimento da forma do tronco, do volume e dimensão dos seus sortimentos, possibilitando o planejamento e o estudo da viabilidade econômica do povoamento.

Segundo Finger et al. (1995), variações na forma do fuste ocorrem em função do espaçamento, tratos culturais, posição sociológica, sítio, idade (seja como um fenômeno evolutivo natural, ou como resultado das várias fases de concorrência relativas às árvores vizinhas), não apresentando uma forma homogênea, mas várias formas geométricas.

Segundo Newnham (1988), existem duas razões para a importância dessa área de estudo. Primeiro, nenhuma teoria desenvolvida explica adequadamente toda a variedade de formas que os troncos das árvores podem assumir. Segundo, e mais importante do ponto de vista prático, uma função de forma é necessária para estimar o volume da árvore em pé para usos múltiplos, sendo, portanto, fundamental para avaliações econômicas e de alta relevância para a tomada de decisão do momento apropriado para intervir na floresta.

Friedl (1989), estudando a dinâmica e prognose da forma dos fustes em povoamentos de Araucaria angustifolia, avaliou o efeito dos fatores idade, sítio e posição sociológica nos fatores de forma naturais. A idade teve um efeito altamente significativo sobre o comportamento da forma das árvores.

Burger et al. (1980) e Machado (1982), ao trabalharem com dados de análise de tronco de Araucárias dominantes, coletados em plantios desde Minas Gerais até o Rio Grande do Sul, ajustaram o polinômio de quinto grau em classes de idade, com amplitude de três anos, e observaram que a qualidade do ajuste aumentou com o avanço da idade. Os autores concluíram que a forma das Araucárias dominantes em plantações torna-se cada vez mais homogênea à medida que a idade aumenta.

Nesse contexto, objetivou-se avaliar o efeito da idade no ajuste de funções de afilamento e no sortimento de uma floresta plantada de Araucaria angustifolia com dados de análise de tronco.

\section{MATERIAL E MÉTODOS}

\subsection{Origem e detalhamento dos dados}

Os dados utilizados nesta pesquisa são de 60 árvores de Araucaria angustifolia de um plantio com 30 anos de idade localizado em Laranjeiras do Sul, Paraná. O plantio foi realizado com espaçamento inicial de $2 \times 2$ m e sofreu 3 desbastes.

Foram amostradas 30 árvores masculinas e 30 femininas, sendo essas selecionadas aleatoriamente de forma a contemplar a variabilidade diamétrica do plantio. 
Tabela 1. Modelos testados para estimar os diâmetros com casca ao longo do tronco $\left(\mathrm{d}_{\mathrm{cc}}\right)$.

Table 1. Tested models to estimate diameters outside bark along of the stem.

\section{Autor/Fonte}

Laar (Schneider \& Silva, 1979)

Laar (modificado) (Schneider \& Silva, 1979)

Schneider (1978)

Schneider (1978) (modificado)

Modelo

Equação

$$
d_{i}=b_{0}+b_{1} d_{s c}+b_{2} I+b_{3} d_{s c} I+\varepsilon_{i}
$$

$$
d_{i}=b_{0}+b_{1} d_{s c}+b_{2} I+b_{3} d_{s c} I+b_{4} h_{i}+\varepsilon_{i}
$$

$$
\ln d_{i}=b_{0}+b_{1} \ln d_{s c}+b_{2} \ln (h I)+\varepsilon_{i}
$$

em que: $d_{1}=$ diâmetro com casca $(\mathrm{cm})$ na altura $h_{\mathrm{i}}(\mathrm{m}) ; \mathrm{d}_{\mathrm{c}}=$ diâmetro sem casca $(\mathrm{cm})$ medido na altura $\mathrm{h}_{\mathrm{f}}(\mathrm{m}) ; \mathrm{h}=$ altura total $(\mathrm{m}) ; \mathrm{I}=$ idade $\left(\right.$ anos); $\mathrm{h}_{\mathrm{i}}=$ altura $(\mathrm{m})$ onde se encontra o diâmetro $\mathrm{d}_{\mathrm{i}}(\mathrm{cm}) ; \ln =$ logaritmo neperiano; $\mathrm{b}_{\mathrm{i}}=$ coeficientes dos modelos; $\varepsilon_{\mathrm{i}}=$ erros aleatórios.

Após a derrubada das árvores selecionadas, 11 discos foram coletados por árvore, nas alturas absolutas $0,1 \mathrm{~m}$ e 1,3 m e nas alturas relativas de $15 \%, 25 \%, 35 \%, 45 \%$, $55 \%, 65 \%, 75 \%, 85 \%$ e $95 \%$ da altura total. Os discos foram secos em estufa industrial para madeira a $60^{\circ} \mathrm{C}$ durante quatro dias. Após a secagem foram lixados e submetidos a técnica de análise de tronco completa (ANATRO), que recupera o crescimento passado da árvore em diâmetro, altura total, volume, obtendo-se também o perfil do tronco em qualquer idade. Para o cálculo dos parâmetros dendrométricos das árvores foi utilizado o programa FlorExel ${ }^{\circledR}$ (OpTimber, 2013).

\subsection{Estimativa dos diâmetros com casca ao longo do tronco nas várias idades}

A análise de tronco permite determinar os diâmetros com casca apenas para a idade em que a árvore foi derrubada. Para obter os diâmetros com casca ao longo do tronco $\left(d_{c c}\right)$ nas demais idades foram testados quatro modelos (Equações 1 a 4), apresentados na Tabela 1. Para aumentar a base de dados e ter uma maior variabilidade de idade, mais oito árvores de Araucaria angustifolia masculinas e femininas com idade variando de 14 a 29 anos, oriundas de plantios na FLONA de Três Barras, Santa Catarina, foram incorporadas a base de dados inicial.

\subsection{Funções de afilamento}

O polinômio de quinto grau é a função de afilamento mais popularmente conhecida no Sul do Brasil e tem sido frequentemente utilizada em inventários florestais quando o objetivo é quantificar multiprodutos e também avaliar a evolução da forma do tronco com o avanço da idade. Isso se deve ao fato da facilidade de ajuste e da boa qualidade que em geral esse modelo proporciona.
Assim sendo, essa função de afilamento (Equação 5) foi utilizada para descrever a forma do tronco das Araucárias e também para estimar os sortimentos.

$$
\frac{d_{i}}{D A P}=b_{0}+b_{1}\left(\frac{h_{i}}{h}\right)+b_{2}\left(\frac{h_{i}}{h}\right)^{2}+b_{3}\left(\frac{h_{i}}{h}\right)^{3}+b_{4}\left(\frac{h_{i}}{h}\right)^{4}+b_{5}\left(\frac{h_{i}}{h}\right)^{5}+\varepsilon_{i}
$$

em que: DAP = diâmetro a altura do peito com casca $(\mathrm{cm}) ; \mathrm{d}_{\mathrm{i}}, \mathrm{hi}, \mathrm{h}, \varepsilon_{\mathrm{i}}, \mathrm{b}_{\mathrm{i}}$ já nominados.

O modelo foi ajustado no Microsoft Excel, usando regressão linear múltipla. Para análise e seleção dos modelos ajustados foram utilizadas as seguintes estatísticas: coeficiente de determinação $\left(\mathrm{R}^{2}\right)$ e do erro padrão da estimativa em porcentagem (Syx\%), além da análise gráfica de resíduos.

\subsection{Influência da idade na forma do tronco}

A influência da idade na forma do tronco de Araucária foi avaliada com as funções de afilamento ajustadas para todos os dados e estratificando-os em classes de idade. Para isso foram construídos gráficos representando as curvas de afilamento e posteriormente aplicado o teste F proposto por Graybill (1976). Esse teste compara a regressão de modelos polinomiais, verificando estatisticamente, a partir da análise de variância e do teste $\mathrm{F}$, se existe diferença entre a soma dos quadrados das regressões ajustadas para cada classe de idade e a soma dos quadrados da regressão ajustada para o conjunto de todas as idades, ou seja, testa a necessidade ou não de desenvolver equações de afilamento, estratificando-se os dados em classes de idade. Graybill (1976) desenvolveu esse teste para verificar a igualdade de um conjunto de equações de regressão, testando a seguinte hipótese de nulidade:

$\mathrm{H} 0: \mathrm{b}_{0}=\mathrm{b}_{1}=\ldots=\mathrm{b}_{\mathrm{n}}$ (as "n" equações são idênticas);

$H 1: b_{i}, \neq b_{j}$, para pelo menos um $i \neq j$ (as "n" equações não são idênticas). 


\subsection{Avaliação dos sortimentos por idade}

Para estimar os volumes por sortimentos nas diferentes classes de idade foi utilizado o módulo de inventário florestal do programa FlorExel ${ }^{\circ}$ (OpTimber, 2013), estimando-se, a partir da função de afilamento ajustada, os volumes totais e por sortimento para as árvores amostradas.

Os sortimentos são definidos com base em diâmetros (com casca) mínimos de uso, denominados de "diâmetro de topo", e no comprimento das toras. Na Tabela 2 estão as classes de sortimento atualmente utilizadas pelas indústrias que operam com matéria-prima de plantios florestais. Essas classes de sortimento foram usadas por Kohler (2013), que trabalhou com a evolução do afilamento do tronco e do sortimento em plantios de Pinus taeda nos estados do Paraná e Santa Catarina.

\subsection{Avaliação dos sortimentos para árvores femininas e masculinas de Araucária}

Considerando-se que a amostragem foi realizada em árvores femininas (30) e masculinas (30), uma análise do sortimento produzido em relação ao sexo foi realizada visando subsidiar decisões de manejo, visto que árvores femininas, além de madeira, produzem também o pinhão, importante produto não madeireiro que pode também ser mais uma opção para agregar renda na atividade com plantações de Araucária.

\section{RESULTADOS E DISCUSSÃO}

\subsection{Estimativa dos diâmetros com casca ao longo do tronco nas várias idades}

Para estimativa do diâmetro com casca ao longo do tronco nas várias idades foi selecionado o modelo de Schneider (1978) modificado, considerando-se que

Tabela 2. Classes de sortimento adaptadas para Araucaria angustifolia.

Table 2. Assortment classes adapted for Araucaria angustifolia.

\begin{tabular}{ccc}
\hline Destinação & $\begin{array}{c}\text { Diâmetro na } \\
\text { ponta fina } \mathbf{( c m})\end{array}$ & $\begin{array}{c}\text { Comprimento } \\
\text { das toras }(\mathbf{m})\end{array}$ \\
\hline Resíduos & 5 & 2,4 \\
\hline Celulose & 8 & 2,4 \\
\hline Serraria 1 & 16 & 3,1 \\
\hline Serraria 2 & 23 & 3,1 \\
\hline Laminação & 35 & 3,1 \\
\hline
\end{tabular}

esse modelo apresentou (Tabela 3) o menor erro padrão de estimativa $\left(\mathrm{S}_{\mathrm{yx}}=2,33 \%\right)$ além de uma distribuição de resíduos mais homogênea (Figura 1).

\subsection{Funções de afilamento}

Os resultados dos ajustes do polinômio de quinto grau para o total e por classes de idade são apresentados na Tabela 4. Observa-se que as equações testadas apresentaram boas estatísticas de ajuste e precisão (exceto na classe de 6 a 10 anos). O erro médio para estimar os diâmetros ao longo do tronco com as equações para as classes de idade a partir de 16 anos foi inferior ao erro da equação geral, de $8,8 \%$, exceto nas duas primeiras classes de idade, constatando-se uma leve vantagem no uso de equações ajustadas por classes de idade. Kohler et al. (2013) ajustaram modelos de afilamento para Pinus taeda e avaliaram a necessidade ou não de desenvolver modelos por classe de idade. Segundo os autores, as equações de afilamento obtidas para as classes de idades apresentaram certa superioridade em relação à equação geral, sugerindo que trabalhar com dados em classes de idade pode proporcionar maior precisão nas estimativas do diâmetro ao longo do tronco.

\subsection{Influência da idade na forma do tronco}

Os resultados do teste proposto por Graybill (1976) estão na Tabela 5, constatando-se que a curva de afilamento ajustada sem o agrupamento é diferente das curvas geradas por classes de idade. Testou-se, então, a igualdade entre as classes de idade, duas a duas, e, como resposta, tem-se que as curvas de afilamento são diferentes para todas as comparações realizadas. Concluindo-se, portanto, que a forma dos fustes altera-se de forma significativa de uma classe de idade para outra e, consequentemente, não é apropriado aplicar uma equação geral a todas as idades.

Como pode ser observado na Figura 2, a forma do fuste das árvores altera-se com a idade, sendo que o afilamento diminui com o avanço da idade, ou seja, os fustes tornam-se mais cilíndricos. Em geral, esses resultados são esperados do ponto de vista biométrico, conforme também já constatado por vários outros autores, dentre os quais Kohler (2013), estudando a evolução da forma para árvores de Pinus taeda, e Friedl (1989), utilizando dados de Araucaria angustifolia. 

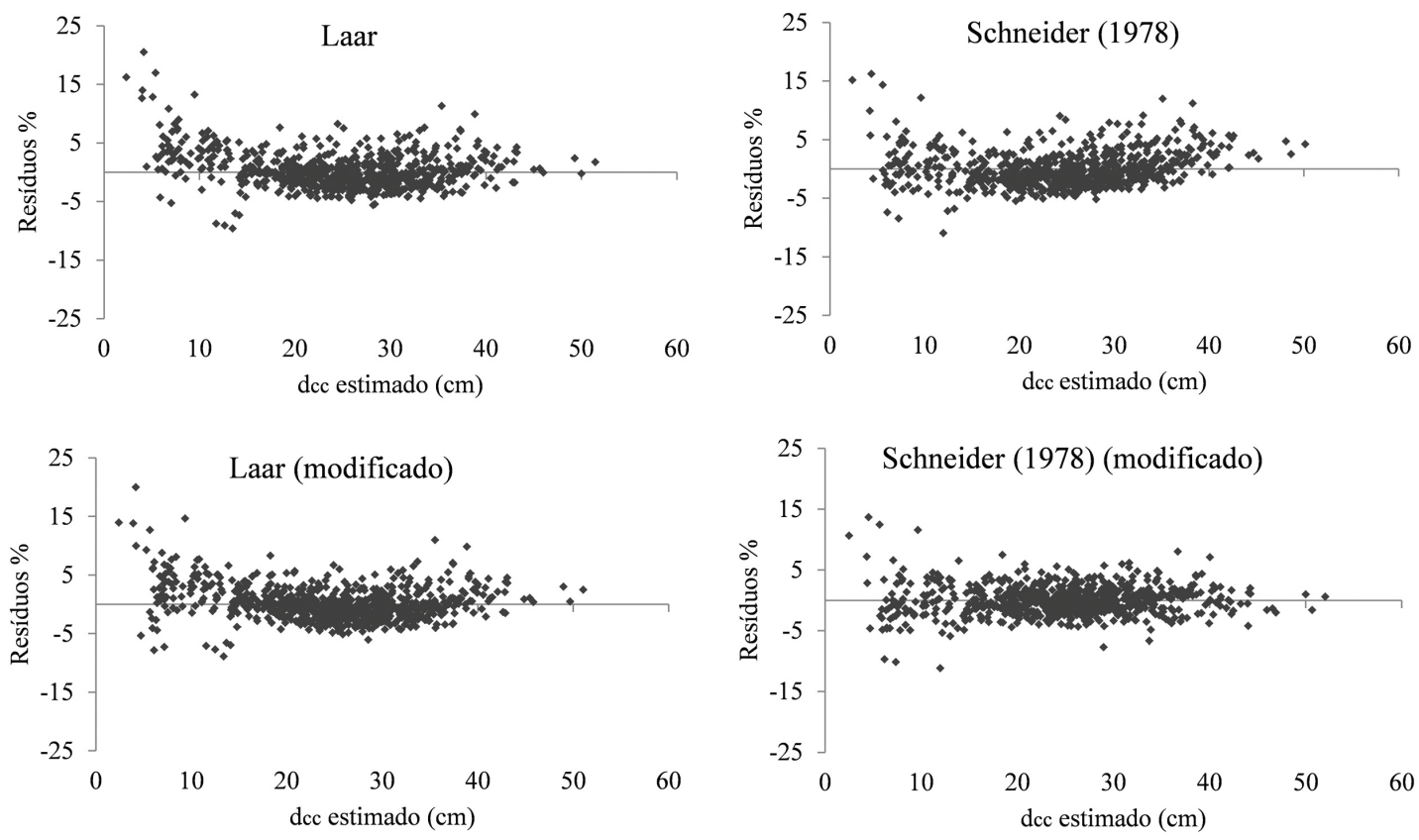

Figura 1. Distribuição dos resíduos para as equações ajustadas para estimativa do diâmetro com casca ao longo do tronco.

Figure 1. Distribution of residuals to equations adjusted to estimate the diameters outside bark along of the stem.

Tabela 3. Coeficientes e estatísticas das equações ajustadas para estimativa do diâmetro com casca ao longo do tronco. Table 3. Coefficients and statistics for the equations adjusted to estimate the diameter outside bark along of the stem.

\begin{tabular}{|c|c|c|c|c|c|c|c|c|}
\hline \multirow{2}{*}{ Autor/Fonte } & \multicolumn{5}{|c|}{ Coeficientes } & \multirow{2}{*}{$\mathbf{R}_{\text {ajust }}^{2}$} & \multirow[b]{2}{*}{$S_{y x}(\mathrm{~cm})$} & \multirow{2}{*}{$\mathrm{S}_{\mathrm{yx}} \%$} \\
\hline & $b_{0}$ & $b_{1}$ & $b_{2}$ & $\mathbf{b}_{3}$ & $\mathbf{b}_{4}$ & & & \\
\hline Laar (Schneider \& Silva, 1979) & $-2,6973$ & 1,6098 & 0,1005 & $-0,0168$ & - & 0,99 & 0,71 & 2,88 \\
\hline $\begin{array}{l}\text { Laar (modificado) (Schneider \& } \\
\text { Silva, 1979) }\end{array}$ & $-2,4473$ & 1,5166 & 0,1362 & $-0,0150$ & $-0,0575$ & 0,99 & 0,69 & 2,79 \\
\hline Schneider (1978) & 0,4674 & 0,9554 & $-0,0338$ & - & - & 0,99 & 0,79 & 3,21 \\
\hline Schneider (1978) (modificado) & 0,4199 & 0,9253 & $-0,0152$ & - & - & 0,99 & 0,57 & 2,33 \\
\hline
\end{tabular}

em que: $b_{i}=$ coeficientes dos modelos; $R_{\text {ajust }}^{2}=$ coeficiente de determinação ajustado; $S_{y x}(\mathrm{~cm})=$ erro padrão de estimativa em centímetros; $\mathrm{S}_{\mathrm{yx}} \%$ = erro padrão de estimativa em porcentagem.

Tabela 4. Coeficientes e estatísticas do polinômio de quinto grau ajustado para estimar o diâmetro nas várias alturas, por classes de idade (anos).

Table 4. Coefficients and statistics for the fifth degree polynomial adjusted to estimate the diameters at various heights, by age classes (years).

\begin{tabular}{|c|c|c|c|c|c|c|c|c|c|}
\hline \multirow{2}{*}{$\begin{array}{l}\text { Classes } \\
\text { de idade }\end{array}$} & \multicolumn{6}{|c|}{ Coeficientes } & \multicolumn{3}{|c|}{ Estatísticas } \\
\hline & $b_{0}$ & $b_{1}$ & $b_{2}$ & $b_{3}$ & $\mathbf{b}_{4}$ & $b_{5}$ & $\mathbf{R}^{2}$ & $S_{y x}(\mathrm{~cm})$ & $\mathrm{S}_{\mathrm{yx}}(\%)$ \\
\hline 6 a 10 & 1,3028 & $-2,5269$ & 6,4158 & $-11,7734$ & 9,7093 & $-3,0531$ & 0,87 & 1,94 & 18,08 \\
\hline 11 a 15 & 1,1854 & $-2,3732$ & 8,0425 & $-15,9933$ & 13,5237 & $-4,3316$ & 0,96 & 1,38 & 9,22 \\
\hline 16 a 20 & 1,1689 & $-2,4262$ & 8,5723 & $-17,0817$ & 15,3580 & $-5,5297$ & 0,97 & 1,36 & 7,32 \\
\hline 21 a 25 & 1,1714 & $-2,7246$ & 10,7705 & $-23,0880$ & 22,8055 & $-8,8826$ & 0,97 & 1,49 & 6,84 \\
\hline 26 a 30 & 1,1728 & $-2,9476$ & 12,7979 & $-29,5003$ & 31,3470 & $-12,800$ & 0,98 & 1,38 & 5,71 \\
\hline Total & 1,1958 & $-2,7513$ & 10,4239 & $-22,0596$ & 21,2510 & $-7,9911$ & 0,96 & 1,71 & 8,85 \\
\hline
\end{tabular}

em que: $b_{i}=$ coeficientes do modelo; $R^{2}=$ coeficiente de determinação; $S_{y x}(\mathrm{~cm})=$ erro padrão de estimativa em centímetros; $\mathrm{S}_{\mathrm{yx}} \%=$ erro padrão de estimativa em porcentagem. 


\subsection{Avaliação dos sortimentos por idade}

Utilizando-se o programa FlorExel ${ }^{\circledR}$ (OpTimber, 2013), os volumes dos sortimentos foram estimados com base nas equações de afilamento ajustadas anteriormente para as classes de idade. Na Tabela 6 observa-se a evolução ou a dinâmica dos volumes de cada sortimento. Volumes de madeira para celulose e energia têm maior representatividade nas idades iniciais, mantendo seu volume quase constante nas idades seguintes. Com oito anos, o povoamento já possui algum volume de madeira para Serraria 1, cujo sortimento médio por árvore aumenta até a idade de 13 anos $\left(0,1042 \mathrm{~m}^{3}\right)$. A partir dessa idade, apresenta volumes constantes e isso decorre do surgimento de toras com diâmetros adequados à classe de sortimento seguinte, Serraria 2 , na qual se obtêm em média $0,6481 \mathrm{~m}^{3}$ de madeira por árvore aos 30 anos do povoamento. Volumes para laminação, ou seja, toras com diâmetro maior que $35 \mathrm{~cm}$ são produzidas somente a partir dos 23 anos de idade.

Na Figura 3 é apresentada a evolução do sortimento com o avanço da idade. Observa-se que as curvas dos volumes para Celulose e Energia mantêm a mesma tendência ao longo dos 30 anos. $\mathrm{O}$ volume de madeira para Serraria 1 é superior ao de Serraria 2 até próximo dos 19 anos, quando passam a ser iguais, e o volume para Serraria 2 torna-se maior que para Serraria 1, aumentando rapidamente até os 30 anos, mostrando a

Tabela 5. Resultados para o teste de Graybill (1976) comparando os ajustes para diferentes classes de idade. Table 5. Results for the Graybill (1976) test comparing the adjustment for different age classes.

\begin{tabular}{ccc}
\hline $\begin{array}{c}\text { Comparações } \\
\text { 6 a } 15 \text { contra as } \\
\text { classes } 11 \text { a } 15 \text { e } 6 \text { a } \\
10 \text { anos }\end{array}$ & 6,29383 & p-valor \\
$\begin{array}{c}11 \text { a } 20 \text { contra as } \\
\text { classes } 16 \text { a } 20 \text { e } 11\end{array}$ & 122,4243 & $1,7 \mathrm{E}-122$ \\
a 15 anos & & \\
$\begin{array}{c}16 \text { a } 25 \text { contra as } \\
\text { classes } 21 \text { a } 25 \text { e } 16\end{array}$ & 51,2074 & $4,07 \mathrm{E}-52$ \\
a 20 anos & & \\
$\begin{array}{c}21 \text { a } 30 \text { contra as } \\
\text { classes } 26 \text { a } 30 \text { e } 21\end{array}$ & 100,3963 & $3,3 \mathrm{E}-102$ \\
a 25 anos & & \\
$\begin{array}{c}\text { Geral (todas as } \\
\text { idades) contra } \\
\text { classes de idade }\end{array}$ & 231,9142 & $0,0 \mathrm{E}+00$ \\
\hline
\end{tabular}

evolução do sortimento e da forma, ou seja, um maior número de toras com bitola superior ou igual a $23 \mathrm{~cm}$.

Figueiredo Filho (1991) estudou a evolução do sortimento para árvores resinadas e não resinadas de Pinus elliottii. $\mathrm{O}$ autor afirma que informações sobre os volumes obtidos em cada idade refletem com precisão a evolução do volume de cada classe de sortimento e do volume total, além de mostrar a participação dos volumes não aproveitáveis comercialmente.

Santos (2006) estudou o crescimento de um plantio de Araucaria angustifolia e encontrou um volume médio por árvore de $0,51 \mathrm{~m}^{3}$ aos 35 anos de idade, volume esse inferior ao obtido neste trabalho, que aos 30 anos já era de $0,9680 \mathrm{~m}^{3}$.

$\mathrm{Na}$ Figura 4 mostra-se percentualmente como se desenvolveu o sortimento nas classes de idade definidas para este estudo. Na classe de idade de 6 a 10 anos,

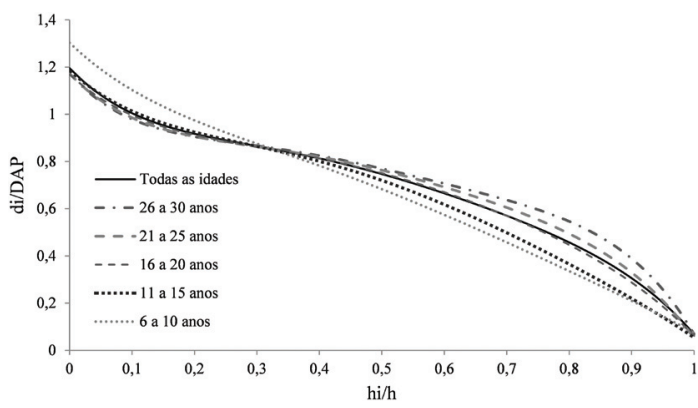

Figura 2. Perfil médio do tronco da amostra (60 árvores) de Araucaria angustifolia por classes de idade e para todas as idades.

Figure 2. Mean stem profile of the Araucaria angustifolia sampling ( 60 trees) by age classes and for all the ages.

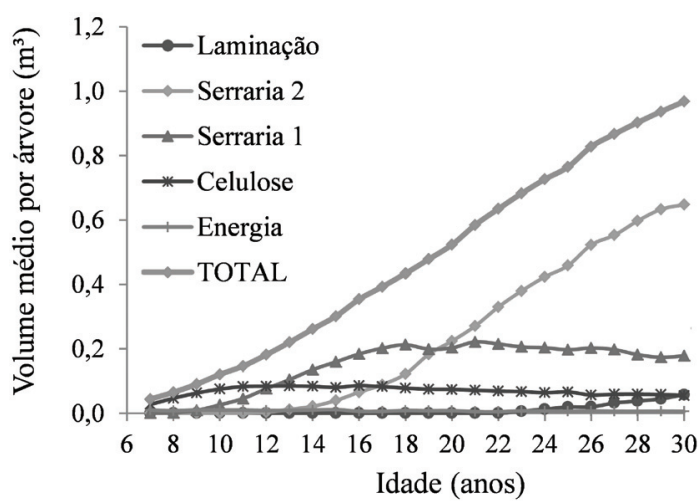

Figura 3. Evolução do volume médio total por árvore $\left(\mathrm{m}^{3}\right)$ e para os sortimentos.

Figure 3. Evolution of the total average volume per tree $\left(\mathrm{m}^{3}\right)$ and for the assortments. 
Tabela 6. Volume médio por árvore $\left(\mathrm{m}^{3}\right)$ para os diferentes sortimentos analisados no estudo.

Table 6. Average volume per tree $\left(\mathrm{m}^{3}\right)$ for different assortments analyzed in the study.

\begin{tabular}{|c|c|c|c|c|c|c|c|c|}
\hline $\begin{array}{l}\text { Idade } \\
\text { (anos) }\end{array}$ & $\begin{array}{l}\text { Lamina- } \\
\text { ção }\end{array}$ & Serraria 2 & Serraria 1 & Celulose & Energia & $\begin{array}{l}\text { Volume } \\
\text { comercial }\end{array}$ & Resíduos & Total \\
\hline 6 & - & - & - & 0,0120 & 0,0070 & 0,0190 & 0,0072 & 0,0262 \\
\hline 7 & - & - & - & 0,0272 & 0,0097 & 0,0368 & 0,0069 & 0,0438 \\
\hline 8 & - & - & 0,0015 & 0,0464 & 0,0081 & 0,0560 & 0,0089 & 0,0649 \\
\hline 9 & - & - & 0,0090 & 0,0627 & 0,0098 & 0,0814 & 0,0088 & 0,0902 \\
\hline 10 & - & - & 0,0260 & 0,0751 & 0,0094 & 0,1105 & 0,0098 & 0,1203 \\
\hline 11 & - & - & 0,0455 & 0,0827 & 0,0097 & 0,1379 & 0,0085 & 0,1464 \\
\hline 12 & - & 0,0030 & 0,0760 & 0,0840 & 0,0084 & 0,1714 & 0,0101 & 0,1815 \\
\hline 13 & - & 0,0119 & 0,1042 & 0,0846 & 0,0089 & 0,2096 & 0,0106 & 0,2202 \\
\hline 14 & - & 0,0215 & 0,1354 & 0,0835 & 0,0097 & 0,2500 & 0,0113 & 0,2613 \\
\hline 15 & - & 0,0396 & 0,1594 & 0,0803 & 0,0111 & 0,2904 & 0,0108 & 0,3012 \\
\hline 16 & - & 0,0653 & 0,1840 & 0,0858 & 0,0061 & 0,3411 & 0,0127 & 0,3538 \\
\hline 17 & - & 0,0888 & 0,2020 & 0,0824 & 0,0058 & 0,3790 & 0,0137 & 0,3927 \\
\hline 18 & - & 0,1220 & 0,2126 & 0,0781 & 0,0087 & 0,4214 & 0,0129 & 0,4343 \\
\hline 19 & - & 0,1831 & 0,1992 & 0,0745 & 0,0079 & 0,4646 & 0,0137 & 0,4783 \\
\hline 20 & - & 0,2241 & 0,2031 & 0,0738 & 0,0082 & 0,5091 & 0,0141 & 0,5232 \\
\hline 21 & - & 0,2708 & 0,2217 & 0,0715 & 0,0050 & 0,5690 & 0,0158 & 0,5847 \\
\hline 22 & - & 0,3300 & 0,2149 & 0,0686 & 0,0047 & 0,6182 & 0,0166 & 0,6348 \\
\hline 23 & 0,0063 & 0,3798 & 0,2059 & 0,0673 & 0,0073 & 0,6665 & 0,0162 & 0,6827 \\
\hline 24 & 0,0126 & 0,4234 & 0,2033 & 0,0638 & 0,0067 & 0,7098 & 0,0167 & 0,7265 \\
\hline 25 & 0,0193 & 0,4587 & 0,1972 & 0,0662 & 0,0059 & 0,7473 & 0,0176 & 0,7649 \\
\hline 26 & 0,0197 & 0,5229 & 0,2024 & 0,0564 & 0,0072 & 0,8086 & 0,0193 & 0,8279 \\
\hline 27 & 0,0318 & 0,5532 & 0,1975 & 0,0589 & 0,0062 & 0,8475 & 0,0194 & 0,8669 \\
\hline 28 & 0,0379 & 0,5973 & 0,1818 & 0,0591 & 0,0058 & 0,8819 & 0,0210 & 0,9029 \\
\hline 29 & 0,0448 & 0,6330 & 0,1736 & 0,0580 & 0,0064 & 0,9158 & 0,0210 & 0,9367 \\
\hline 30 & 0,0577 & 0,6481 & 0,1781 & 0,0551 & 0,0057 & 0,9447 & 0,0233 & 0,9680 \\
\hline
\end{tabular}

quase todo volume das árvores é destinado a Celulose e Energia e apenas 12\% do volume para Serraria 1. Já na classe de 11 a 15 anos, a maior porcentagem do volume passa a ser para Serraria $1(49,1 \%)$ e nessa classe já aparece madeira destinada a Serraria 2 (7,2\%). Ao longo dos anos, a porcentagem para Serraria 2 aumenta, chegando a $67,2 \%$ na classe de 26 a 30 anos. Para laminação, o porcentual ainda é pequeno, iniciando-se na classe de 21 a 25 anos com apenas 1,2\%, no entanto, na classe 26 a 30 anos esse sortimento já é de 4,4\%. Provavelmente essa porcentagem deve aumentar à medida que o povoamento fica mais velho e as toras migram nas classes de sortimento.

Figueiredo Filho (1991) encontrou, para um plantio de Pinus elliottii em Telêmaco Borba, estado do Paraná, um volume médio por árvore de $0,345 \mathrm{~m}^{3}$ aos 15 anos, e nessa idade $34 \%$ da madeira era adequada para celulose e $57 \%$ para serraria. Todavia, aos 23 anos, o volume médio evoluiu para $0,727 \mathrm{~m}^{3}$, dos quais, $48 \%$ podiam ser laminados.

\subsection{Análise do sortimento para árvores masculinas e femininas de Araucária}

A evolução do volume total médio das árvores masculinas e femininas pode ser observada na Figura 5. As árvores femininas apresentaram maior crescimento que as árvores masculinas em todo o seu desenvolvimento, sendo que aos 30 anos as árvores femininas possuíam em média $0,9975 \mathrm{~m}^{3} \mathrm{e}$ as masculinas, $0,9384 \mathrm{~m}^{3}$ de madeira.

Na Figura 6 é mostrada em porcentagem como se desenvolveu o sortimento nas classes de idade determinadas para este estudo. Observa-se que as árvores masculinas apresentam maiores porcentagens de volume nos sortimentos Energia, Celulose e Serraria 1, o que evidencia o crescimento mais lento dessas 

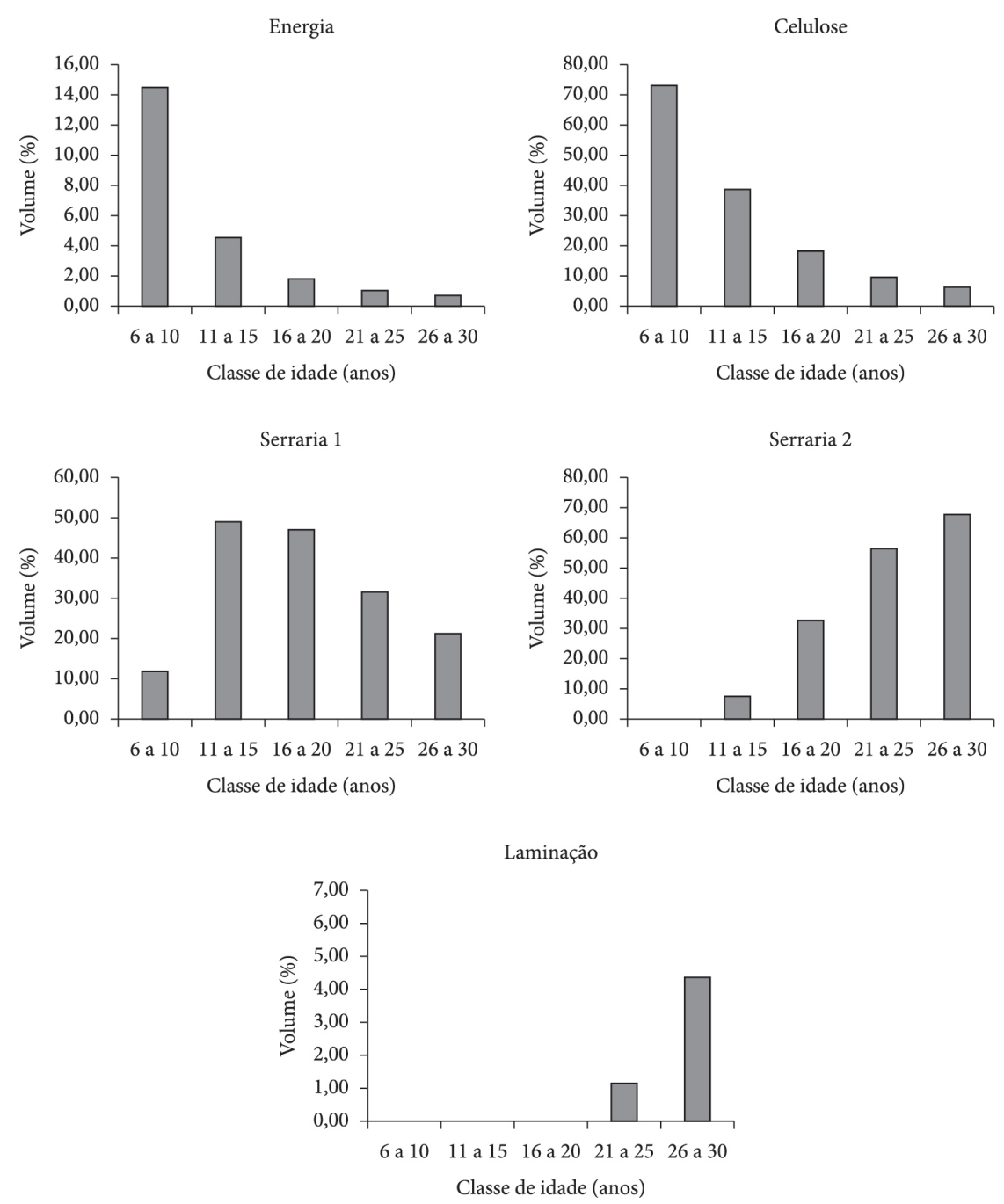

Figura 4. Volume dos sortimentos em porcentagem nas classes de idade.

Figure 4. Assortments volumes in percentage by age classes.

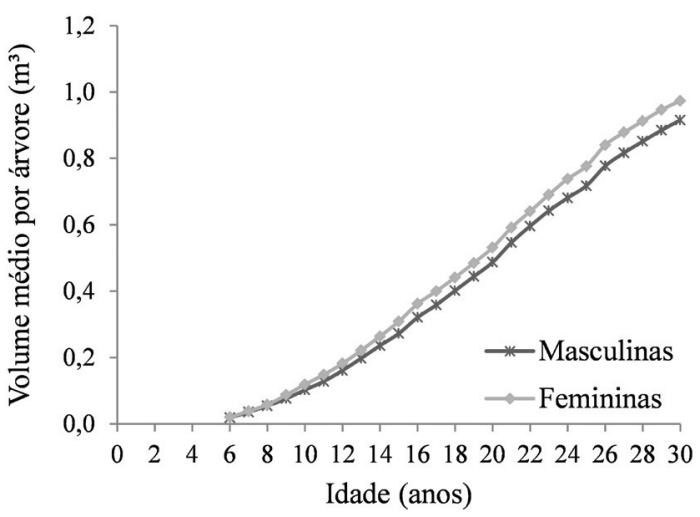

Figura 5. Crescimento médio em volume para árvores masculinas e femininas de Araucária.

Figure 5. Average volume growth for male and female trees of Araucaria. árvores, se comparado com o das femininas, que já apresentavam diâmetros maiores e adequados aos sortimentos mais nobres em uma mesma idade. A maior diferença é observada para a classe de idade de 26 a 30 anos, na qual se tem 6,44\% do volume para laminação em árvores femininas e 2,12\% em árvores masculinas.

Kohler (2013) avaliou o sortimento por classe de idade em plantios de Pinus taeda estabelecidos nos estados do Paraná e Santa Catarina. A autora estimou volumes para laminação $(\mathrm{d}>35 \mathrm{~cm})$ a partir da classe de idade de 13 a 15 anos, representando 1,25\% do volume total para plantios no estado de Santa Catarina e 4,26\% no estado do Paraná. 

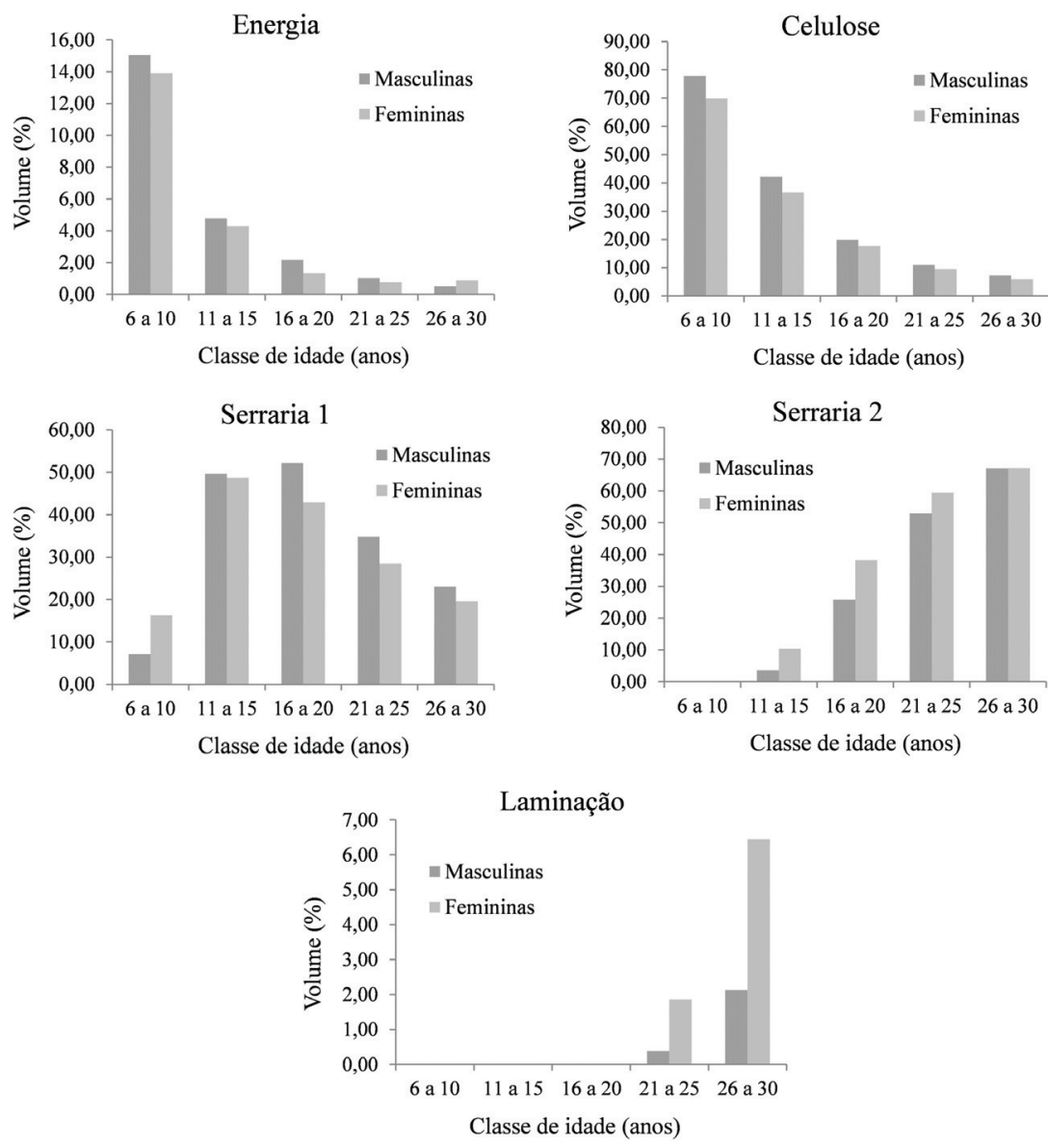

Figura 6. Volume dos sortimentos em porcentagem nas classes de idade de árvores femininas e masculinas. Figure 6. Assortment volumes in percentage in age classes of male and female trees.

\section{CONCLUSÃO}

O teste de Graybill (1976) mostrou que uma única equação de afilamento não deveria ser ajustada para representar várias idades. Assim, o ajuste de funções de afilamento em classes de idade pode gerar melhores estimativas. Como esperado, a forma do fuste das Araucárias melhora significativamente de uma classe de idade para outra.

Aos 30 anos de idade, em média, o povoamento produz cerca de um metro cúbico por árvore, sendo que as árvores femininas apresentam maior crescimento do que as árvores masculinas e isso foi observado ao longo dos 30 anos de desenvolvimento do plantio amostrado.

Sortimentos mais nobres (toras com diâmetros com casca maiores que $23 \mathrm{~cm}$ na ponta fina) são produzidos no povoamento entre 11 e 15 anos de idade, representando $7,17 \%$ do volume total. Dessa forma fica evidente o bom crescimento da Araucária, sugerindo que essa espécie, tão valorizada alguns anos atrás, poderia voltar ao mercado com novos plantios.

\section{STATUS DA SUBMISSÃO}

Recebido: 26 fev., 2014

Aceito: 28 out., 2014

\section{AUTOR(ES) PARA CORRESPONDÊNCIA}

Afonso Figueiredo Filho

Departamento de Engenharia Florestal, Universidade Estadual do Centro-Oeste UNICENTRO, CEP 84500-000, Irati, PR, Paraná, Brasil

e-mail: afigfilho@gmail.com 


\section{REFERENNCIAS}

Burger D, Machado AS, Hosokawa RT. Estudo do desenvolvimento da forma de Araucaria angustifolia com relação a idade. In: Anais do Encontro da International Union for Forestry Research Organization (IUFRO); 1979; Curitiba. Curitiba: IUFRO; 1980. p. 320-329.

Campos JCC, Leite HG. Mensuração florestal: perguntas e respostas. 2. ed. Viçosa: UFV; 2006.

Figueiredo Filho A. Influência da resinagem no crescimento de Pinus elliottii Engelm. var. elliottii e sua avaliação econômica [tese]. Curitiba: Setor de Ciências Agrárias, Universidade Federal do Paraná; 1991.

Finger CAG, Zanon MLB, Schneider PR, Klein JEM, Coelho MCB. Funções de forma para Eucalyptus dunnii Maiden implantados na Depressão Central e Encosta do Sudeste do Rio Grande do Sul. Ciência Rural 1995; 25(3): 399-403. http://dx.doi.org/10.1590/S0103-84781995000300012.

Friedl RA. Dinâmica e prognose da forma dos fustes em povoamentos plantados de Araucaria angustifolia (Bert.) O. Ktze [dissertação]. Curitiba: Setor de Ciências Agrárias, Universidade Federal do Paraná; 1989.

Graybill F. A theory and application of the linear model. Duxbury Press; 1976.

Handro W, Ferreira CM. Araucaria (Araucaria spp.). In: Bajaj Y. Biotechnology in agriculture and forestry. Heidelberg: Springer-Verlag; 1986. p. 310-315.

Husch B, Beers TW, Kershaw JA Jr. Forest mensuration. 4. ed. Hoboken: John Wiley \& Sons; 2003.

Kohler SV, Koehler HS, Figueiredo Filho A. Modelo de afilamento para Pinus taeda por classe de idade. Floresta e Ambiente 2013; 20(4): 470-479.

Kohler SV. Evolução do afilamento do tronco e do sortimento em plantios de Pinus taeda nos estados do Paraná e Santa Catarina [dissertação]. Curitiba: Setor de Ciências Agrárias, Universidade Federal do Paraná; 2013.

Machado SA. Influência da idade na forma do fuste de Araucaria angustifolia em plantações no Brasil. In: Anais do $5^{\circ}$ Seminário sobre atualidades e perspectivas florestais: o uso de funções de forma de tronco em estudo de volumetria de espécies florestais; 1982; Curitiba. Curitiba: EMBRAPA; 1982. p. 51-55.

Môra R. Influência da forma de ajuste e do comprimento das seções no desempenho de funções de afilamento [dissertação]. Jeronimo Monteiro: Universidade Federal do Espírito Santo; 2011.

Newnham RM. A variable-form taper function. Chalk River: Petawawa National Forestry Institute; 1988. 33 p. Information Report no. 83.

OpTimber. FlorExel: Forestry Functions for Microsoft Excel, version 3.11. Curitiba: OpTimber Otimização e Informática; 2013. Available from: www.optimber.com.br.

Queiroz D, Machado SA, Figueiredo Filho A, Arce JE, Koehler HS. Identidade de modelos em funções de afilamento para Mimosa scabrella Bentham em povoamentos nativos da região metropolitana de Curitiba/PR. Floresta 2008; 38(2): 339-349. http://dx.doi.org/10.5380/rf.v38i2.11629.

Reitz R, Klein RM. Araucariáceas. Itajaí; 1966. Flora Ilustrada Catarinense.

Sanquetta CR, Pizatto W, Péllico Netto S, Figueiredo Filho A, Eisfeld RL. Estrutura vertical de um fragmento de Floresta Ombrófila Mista no Centro-sul do Paraná. Floresta 2002; 32(2): 267-276.

Santos WC. Análise de características dendrométricas e fatores edáficos no crescimento e produção de um povoamento de Araucaria angustifolia (Bert.) O. Ktze [dissertação]. Curitiba: Setor de Ciências Agrárias, Universidade Federal do Paraná; 2006.

Schneider PR, Silva JA. Teste de equações para predizer a espessura de casca e do fator K para acácia-negra (Acacia mearnsii De Wild.). Floresta 1979; 10(2): 5-11.

Schneider PR. Modelo de equação e tabelas para avaliar o peso de casca de acácia-negra, Acacia mearnsii de Wild [dissertação]. Curitiba: Universidade Federal do Paraná; 1978.

Zanon MLB. Crescimento da Araucaria angustifolia (Bertol.) Kuntze diferenciado por dioicia [tese]. Santa Maria: Setor de Ciências Agrárias, Universidade Federal de Santa Maria; 2007. 